TAIWANESE JOURNAL OF MATHEMATICS

Vol. 15, No. 4, pp. 1487-1495, August 2011

This paper is available online at http://www.tjm.nsysu.edu.tw/

\title{
DIAMETER PRESERVING MAPPINGS BETWEEN FUNCTION ALGEBRAS
}

\author{
Juan J. Font and Maliheh Hosseini
}

\begin{abstract}
In this paper we study the behaviour of linear diameter preserving mappings when defined between subalgebras of continuous functions. Namely, we obtain a representation of such mappings as the sum of a weighted composition operator and a linear functional on, at least, the Choquet boundaries of the algebras under consideration. In particular, we give a complete description when we consider several classical function algebras.
\end{abstract}

\section{INTRODUCTION}

The study of linear operators defined between spaces of continuous functions which leave a given set, norm, function or relation invariant has received (and receives) considerable attention in the mathematical literature.

Within this context, Györy and Molnár ([9]) introduced a new kind of linear operators based on the preservation of the diameter of the range of the functions. Namely, they considered linear surjective diameter preserving mappings defined between $C(X)$-spaces, where $X$ is compact and first countable, and showed that such operators can be written as the sum of a weighted composition operator and a linear functional. These results were extended, independently, by González and Uspenskij ([8]) and by Cabello ([5]) by removing the hypothesis of first countability. Since then, several authors have extended the above results to several contexts; for example, Rao and Roy ([10]) obtained analogue characterizations for spaces of vector-valued affine functions, Font and Sanchis $([6,7])$ for certain subspaces of real-valued continuous functions, and Aizpuru and Tamayo ([2]) for spaces of vector-valued continuous functions. Other papers dealing with this topic are [1], [3] and [4].

Received December 12, 2009, accepted February 24, 2010.

Communicated by Bor-Luh Lin.

2000 Mathematics Subject Classification: Primary 47B38; Secondary 46J10, 47B33.

Key words and phrases: Diameter preserving maps, Function algebras, Choquet boundary, Extreme point.

Research of J. J. Font was partially supported by the Spanish Ministry of Science and Education (Grant number MTM2008-04599), and by Bancaixa (Projecte P1-1B2008-26). 
In this paper we study the behaviour of linear diameter preserving mappings when defined between subalgebras of continuous functions and, thus, we extend their scope of application to wider contexts.

We first address the case when the underlying spaces are compact. Namely, we obtain, under certain assumptions, a representation for linear diameter preserving mappings between function algebras as the sum of a weighted composition operator and a linear functional on, at least, their Choquet boundaries. We then prove that this result is true for several classical function algebras, including real-valued function algebras, (little) Lipschitz algebras and the algebras of $n$-times continuously differentiable functions, of continuous functions of bounded variation and of absolutely continuous functions.

In the second part of the paper we study the locally compact case.

\section{Preliminaries}

Let $X$ be a locally compact (Hausdorff) space and $X_{\infty}=X \cup\{\infty\}$ be its one point compactification. We denote by $C_{0}(X)$ the algebra of all scalar-valued continuous functions on $X$ vanishing at infinity endowed with the supremum norm $\|\cdot\|_{\infty}$.

A linear subspace $A$ of $C_{0}(X)$ is called a function space on $X$ if $A$ separates strongly the points of $X$, i.e., for each $x, y \in X$ with $x \neq y$, there exists $f \in A$ with $f(x) \neq f(y)$, and for each $x \in X$, there exists $f \in A$ with $f(x) \neq 0$. When $X$ is compact, we shall assume that all function spaces on $X$ contain the set of all constant functions, denoted by $C$.

A function space $A$ on $X$ is called a function algebra on $X$ if it is an algebra. A function algebra $A$ on $X$ is a Banach function algebra on $X$ if it is a Banach algebra with respect to a certain norm. For a Banach function algebra $A$ and $f \in A$, we denote the maximal ideal space of $A$ and the Gelfand transform of $f$ by $M_{A}$ and $\hat{f}$, respectively.

The Choquet boundary, $C h(A)$, of a function space $A$ on $X$ is the set of all $x \in X$ for which $\delta_{x}$, the evaluation homomorphism at $x$, is an extreme point of the unit ball of the dual space of $\left(A,\|.\|_{\infty}\right)$. If $x, x^{\prime} \in C h(A)$, then we define $\delta_{x, x^{\prime}}:=\delta_{x}-\delta_{x^{\prime}}$.

For any function $f \in C_{0}(X), \operatorname{diam}(f)$ denotes the diameter of the range of $f$. For two function spaces $A$ and $B$, a linear map $T: A \rightarrow B$ is called diameter preserving if $\operatorname{diam}(f)=\operatorname{diam}(T f)$ for all $f \in A$.

For a function space $A$ on a compact space $X, A_{d}$ stands for the quotient space $A / C$ endowed with the diameter norm, $\|\pi(f)\|:=\operatorname{diam}(f)$ for all $f \in A$, where $\pi$ is the quotient map $\pi: A \rightarrow A / C$, and $A_{d}^{*}$ its dual space. Moreover, we denote the unit ball of the dual space $A_{d}^{*}$ by $\mathcal{B}_{A_{d}^{*}}$ and its set of extreme points by $\operatorname{ext}\left(\mathcal{B}_{A_{d}^{*}}\right)$. 


\section{The Compact Case}

\section{Theorem 3.1.}

(i) Let $A$ and $B$ be (complex or real-valued) function algebras on compact spaces $X$ and $Y$, respectively, such that $\operatorname{ext}\left(\mathcal{B}_{A_{d}^{*}}\right)=\left\{\alpha \delta_{x, x^{\prime}}: x, x^{\prime} \in C h(A), x \neq\right.$ $\left.x^{\prime}, \alpha \in \mathbb{T}\right\}$. Then, for a linear surjective diameter preserving map $T: A \rightarrow$ $B$, there are a subset $Y_{0}$ of $C h(B)$, a continuous bijective map $\psi: Y_{0} \rightarrow$ $C h(A)$, a scalar $\lambda \in \mathbb{T}$ and a linear functional $L: A \rightarrow \mathbb{C}$ such that, for all $f \in A$ and $y \in Y_{0}, T f(y)=\lambda f(\psi(y))+L(f)$.

(ii) If, in addition, $\operatorname{ext}\left(\mathcal{B}_{B_{d}^{*}}\right)=\left\{\alpha \delta_{y, y^{\prime}}: y, y^{\prime} \in C h(B), y \neq y^{\prime}, \alpha \in \mathbb{T}\right\}$, then $Y_{0}=C h(B)$ and $\psi$ is a homeomorphism from $C h(B)$ onto $C h(A)$.

(iii) If $A$ and $B$ are Banach function algebras in (ii), then $\psi$ can be extended to a homeomorphism $\tilde{\psi}$ from $M_{B}$ onto $M_{A}$ such that, for each $f \in A$, $\widehat{T f} \equiv \lambda \hat{f} \circ \tilde{\psi}+L(f)$ on $M_{B}$.

Proof. (i) It is apparent that $T$ induces a linear isometry $T_{d}: A_{d} \rightarrow B_{d}$ under the diameter norm defined by $T_{d}(\pi(f))=\pi(T f)$ for all $f \in A$. Hence the adjoint of $T$, $T_{d}^{*}: B_{d}^{*} \rightarrow A_{d}^{*}$, is a linear bijective isometry and, so, $T_{d}^{*}\left(\operatorname{ext}\left(\mathcal{B}_{B_{d}^{*}}\right)\right)=\operatorname{ext}\left(\mathcal{B}_{A_{d}^{*}}\right)$. Consequently, $T_{d}^{*-1}\left(\delta_{x_{1}, x_{2}}\right) \in \operatorname{ext}\left(\mathcal{B}_{B_{d}^{*}}\right)$, since, by hypothesis, $\delta_{x_{1}, x_{2}} \in \operatorname{ext}\left(\mathcal{B}_{A_{d}^{*}}\right)$ for each $x_{1}, x_{2} \in C h(A)$.

Let us define the set $C h(A)_{2}:=\left\{\left\{x, x^{\prime}\right\}: x, x^{\prime} \in C h(A), x \neq x^{\prime}\right\}$. Thus, since $A$ is a function algebra, we can define an injective map $\Phi: C h(A)_{2} \rightarrow C h(B)_{2}$ by $\Phi\left\{x_{1}, x_{2}\right\}:=\operatorname{supp}\left(T_{d}^{*^{-1}}\left(\delta_{x_{1}, x_{2}}\right)\right)$.

Let $x \in C h(A)$. We now show that, for each pair of different points $x_{1}, x_{2} \in$ $C h(A)$ distinct from $x, \operatorname{card}\left(\Phi\left\{x_{1}, x\right\} \cap \Phi\left\{x_{2}, x\right\}\right)=1$. Since $\delta_{x_{1}, x}-\delta_{x, x_{2}}=$ $\delta_{x_{1}, x_{2}} \in \operatorname{ext}\left(\mathcal{B}_{A_{d}^{*}}\right)$, then $T_{d}^{*^{-1}}\left(\delta_{x_{1}, x}\right)-T_{d}^{*^{-1}}\left(\delta_{x, x_{2}}\right) \in \operatorname{ext}\left(\mathcal{B}_{B_{d}^{*}}\right)$. On the other hand, we know that the set $\left\{\delta_{y}: y \in C h(B)\right\}$ is linearly independent in $B^{*}$ because $B$ is a function algebra. Then, since $\operatorname{ext}\left(\mathcal{B}_{B_{d}^{*}}\right)$ is included in the set $\left\{\alpha \delta_{y, y^{\prime}}: y, y^{\prime} \in C h(B), y \neq y^{\prime}, \alpha \in \mathbb{T}\right\}$ (by a similar argument to [7, Theorem 1]) and $\Phi$ is injective, it follows that $\operatorname{card}\left(\Phi\left\{x_{1}, x\right\} \cap \Phi\left\{x_{2}, x\right\}\right)=1$. Let $\varphi(x)$ be the unique point in this intersection.

We next prove that $\varphi(x)$ is independent of $x_{1}, x_{2} \in C h(A)$. To see this, we must show that $\varphi(x) \in \Phi\left\{x, x^{\prime}\right\}$ for each $x^{\prime} \in C h(A)$ distinct from $x$. Contrary to what we claim, choose $x_{3} \in C h(A) \backslash\left\{x, x_{1}, x_{2}\right\}$ such that $\varphi(x)$ does not belong to $\Phi\left\{x, x_{3}\right\}$. From the above argument, there exist distinct points $y_{1}, y_{2} \in$ $C h(B) \backslash\{\varphi(x)\}$ with $\Phi\left\{x_{1}, x\right\}=\left\{\varphi(x), y_{1}\right\}$ and $\Phi\left\{x_{2}, x\right\}=\left\{\varphi(x), y_{2}\right\}$. Again, by the same argument, we have $\Phi\left\{x_{3}, x\right\}=\left\{y_{1}, y_{2}\right\}$. On the other hand, by the linear independence of the set $\left\{\delta_{y}: y \in C h(B)\right\}$,

$$
T_{d}^{*^{-1}}\left(\delta_{x_{1}}-\delta_{x_{2}}\right)=T_{d}^{*^{-1}} \delta_{x_{1}, x}-T_{d}^{*^{-1}} \delta_{x, x_{2}}=\beta \delta_{y_{1}, \varphi(x)}-\beta \delta_{\varphi(x), y_{2}}=\beta \delta_{y_{1}, y_{2}},
$$


for some $\beta \in \mathbb{T}$. Then $\Phi\left\{x_{1}, x_{2}\right\}=\left\{y_{1}, y_{2}\right\}$, which is a contradiction with the injectivity of $\Phi$. Thus $\varphi(x)$ is independent of $x_{1}, x_{2} \in C h(A)$.

Therefore, there exist $\beta \in \mathbb{T}$ and an injective map $\varphi: C h(A) \rightarrow C h(B)$ such that $T_{d}^{*^{-1}} \delta_{x, x^{\prime}}=\beta \delta_{\varphi(x), \varphi\left(x^{\prime}\right)}$ for each $x, x^{\prime} \in C h(A)$. Let $\psi:=\varphi^{-1}: Y_{0} \rightarrow$ $C h(A)$, where $Y_{0}=\varphi(C h(A))$. Then, $\psi$ is a bijective map such that for all $y, y^{\prime} \in Y_{0}, T_{d}^{*}\left(\delta_{y}-\delta_{y^{\prime}}\right)=\lambda\left(\delta_{\psi(y)}-\delta_{\psi\left(y^{\prime}\right)}\right)$, where $\lambda=\bar{\beta}$. Hence, for each $f \in A$ and $y, y^{\prime} \in Y_{0}, T f(y)-T f\left(y^{\prime}\right)=\lambda\left(f(\psi(y))-f\left(\psi\left(y^{\prime}\right)\right)\right)$. Now, by fixing $y^{\prime} \in Y_{0}$, define a linear functional $L: A \rightarrow \mathbb{C}$ by $L(f)=-\lambda f\left(\psi\left(y^{\prime}\right)\right)+T f\left(y^{\prime}\right)$ for all $f \in A$. Thus, for all $f \in A$ and $y \in Y_{0}, T f(y)=\lambda f(\psi(y))+L(f)$.

Finally we prove that $\psi$ is continuous. Fix $y_{0} \in Y_{0}$ and let $\left(y_{i}\right)_{i}$ be a net in $Y_{0}$ convergent to $y_{0}$ such that $\left(\psi\left(y_{i}\right)\right)_{i}$ converges to $x_{0}$ in $X$. It is enough to show that $x_{0}=\psi\left(y_{0}\right)$. Contrary to what we claim, choose a neighborhood $U$ of $\psi\left(y_{0}\right)$ that $x_{0} \in X \backslash U$. Since $\psi\left(y_{0}\right) \in C h(A)$, there exists a function $f \in \bar{A}$, where $\bar{A}$ is the closure of $A$ in $\left(C(X),\|\cdot\|_{\infty}\right)$, such that $\|f\|_{\infty}=f\left(\psi\left(y_{0}\right)\right)=1$ and $|f|<\frac{1}{4}$ on $X \backslash U$. Choose $g \in A$ such that $\|f-g\|_{\infty}<\frac{1}{4}$. Then, in particular, $\left|g\left(\psi\left(y_{0}\right)\right)\right|>\frac{3}{4}$ and $|g|<\frac{1}{2}$ on $X \backslash U$. We can consider $i_{0}$ such that, for all $i \geq i_{0}$, $\left|T g\left(y_{i}\right)-T g\left(y_{0}\right)\right|<\frac{1}{4}$. On the other hand, since

$$
\begin{aligned}
\lim _{i \rightarrow \infty}\left|T g\left(y_{i}\right)-T g\left(y_{0}\right)\right| & =\lim _{i \rightarrow \infty}\left|g\left(\psi\left(y_{i}\right)\right)-g\left(\psi\left(y_{0}\right)\right)\right| \\
& =\left|g\left(x_{0}\right)-g\left(\psi\left(y_{0}\right)\right)\right| \geq \frac{3}{4}-\left|g\left(x_{0}\right)\right| \geq \frac{3}{4}-\frac{1}{2}=\frac{1}{4},
\end{aligned}
$$

then, for a sufficiently large index $i,\left|T g\left(y_{i}\right)-T g\left(y_{0}\right)\right| \geq \frac{1}{4}$ and this is a contradiction. Thus $x_{0}=\psi\left(y_{0}\right)$ and $\psi$ is continuous.

(ii) Let us take $y \in C h(B)$ and choose an element $y^{\prime} \in Y_{0}$ different from $y$. Then $\delta_{y, y^{\prime}} \in \operatorname{ext}\left(\mathcal{B}_{B_{d}^{*}}\right)$ and, so, there exist $x, x^{\prime} \in C h(A)$ such that $T_{d}^{*^{-1}} \delta_{x, x^{\prime}}=$ $\bar{\lambda} \delta_{y, y^{\prime}}$. On the other hand, $T_{d}^{*^{-1}} \delta_{x, x^{\prime}}=\bar{\lambda} \delta_{\varphi(x), \varphi\left(x^{\prime}\right)}$. Thus, as above, it follows that $\delta_{y, y^{\prime}}=\delta_{\varphi(x), \varphi\left(x^{\prime}\right)}$. Then it is apparent that $y=\varphi(x)$. Therefore, $y \in Y_{0}$, i.e., $Y_{0}=C h(B)$.

Finally we prove that $\psi$ is a homeomorphism. As above, for $T_{d}^{*-1}: A_{d}^{*} \rightarrow B_{d}^{*}$ we can find a continuous bijective map $\psi^{\prime}: C h(A) \rightarrow C h(B)$ such that $T_{d}^{*-1}\left(\delta_{x}-\right.$ $\left.\delta_{x^{\prime}}\right)=\bar{\lambda}\left(\delta_{\psi^{\prime}(x)}-\delta_{\psi^{\prime}\left(x^{\prime}\right)}\right)$ for all $x, x^{\prime} \in C h(A)$. Also for all $y, y^{\prime} \in C h(B)$ we have $T_{d}^{*}\left(\delta_{y}-\delta_{y^{\prime}}\right)=\lambda\left(\delta_{\psi(y)}-\delta_{\psi\left(y^{\prime}\right)}\right)$. Then $\delta_{\psi^{\prime}(\psi(y))}-\delta_{\psi^{\prime}\left(\psi\left(y^{\prime}\right)\right)}=\delta_{y}-\delta_{y^{\prime}}$ and $\delta_{\psi\left(\psi^{\prime}(x)\right)}-\delta_{\psi\left(\psi^{\prime}\left(x^{\prime}\right)\right)}=\delta_{x}-\delta_{x^{\prime}}$ for all $x, x^{\prime} \in C h(A)$ and $y, y^{\prime} \in C h(B)$. These equations imply that $\psi^{\prime}$ is the converse of $\psi$ and, so, $\psi$ is a homeomorphism.

(iii) Let $f \in A$. By (ii), there are a homeomorphism from $C h(B)$ onto $C h(A)$, a scalar $\lambda \in \mathbb{T}$ and a linear functional $L: A \rightarrow \mathbb{C}$ such that the equation $T f=$ $\lambda f \circ \psi+L(f)$ holds on $C h(B)$. Since $C h(B)$ is a boundary for $B$, there is unique element $g_{f} \in A$ such that $g_{f}=f \circ \psi$ on $C h(B)$. Then the map $S: A \rightarrow B$ 
defined by $S f=g_{f}$ is a homomorphism from $A$ onto $B$. Hence the restriction of $S^{*}$, the adjoint of $S$, to $M_{B}$ is the desired extension of $\psi$. Also for each $f \in A$ and $y \in M_{B}$ we have

$$
\widehat{T f}(y)=y(T f)=y(\lambda S f+L(f))=\lambda \hat{f}\left(S^{*} y\right)+L(f)=\lambda \hat{f}(\tilde{\psi}(y))+L(f) .
$$

Remark 3.2. It is interesting to compare Theorem 3.1.(iii) with [9, Example 1], which shows that each homeomorphism between the Choquet boundaries of Banach function algebras cannot be always extended to their maximal ideal spaces.

The following proposition shows that there are a lot of function algebras that satisfy the assumptions in Theorem 3.1.

Proposition 3.3. Let $A$ be a (complex or real-valued) function space on a compact space $X$ which is dense in $\left(C(X),\|\cdot\|_{\infty}\right)$. Then $\operatorname{ext}\left(\mathcal{B}_{A_{d}^{*}}\right)=\left\{\alpha \delta_{x, x^{\prime}}\right.$ : $\left.x, x^{\prime} \in X, x \neq x^{\prime}, \alpha \in \mathbb{T}\right\}$.

Proof. As before, $\mathcal{B}_{A_{d}^{*}}$ and $\mathcal{B}_{C(X)_{d}^{*}}$ denote the unit ball of the dual spaces of the quotient spaces of $A / C$ and $C(X) / C$ with respect to the diameter norm, respectively. We claim that the restriction map $\tau: \mathcal{B}_{C(X)_{d}^{*}} \rightarrow \mathcal{B}_{A_{d}^{*}}$ is a bijective isometry. Clearly, since $A$ is dense in $\left(C(X),\|\cdot\|_{\infty}\right)$, then $\tau$ is an injective isometry. Now, in order to obtain the surjectivity of $\tau$, let $\varphi \in \mathcal{B}_{A_{d}^{*}}$. For $f \in C(X)$, there is a sequence $\left\{f_{n}\right\} \subseteq A$ with $\left\|f_{n}-f\right\|_{\infty} \rightarrow 0$. In particular, $\operatorname{diam}\left(f_{n}-f\right) \rightarrow 0$ and, so, $\pi\left(f_{n}\right) \rightarrow \pi(f)$. Thus $\left|\varphi\left(\pi\left(f_{n}\right)\right)-\varphi\left(\pi\left(f_{m}\right)\right)\right| \leq\left\|\pi\left(f_{n}\right)-\pi\left(f_{m}\right)\right\| \rightarrow 0$ and, then, $\left\{\varphi\left(\pi\left(f_{n}\right)\right)\right\}$ is a Cauchy sequence in $\mathbb{C}$. Take $\tilde{\varphi}(f)=\lim _{n \rightarrow \infty} \varphi\left(\pi\left(f_{n}\right)\right)$. It is easily checked that this definition is independent of the choice of the sequence $\left\{f_{n}\right\}$. Thus, due to the density of $A$ in $\left(C(X),\|\cdot\|_{\infty}\right),\|\tilde{\varphi}\|=\|\varphi\|$ and $\tilde{\varphi} \in \mathcal{B}_{C(X)_{d}^{*}}$; moreover, $\left.\tilde{\varphi}\right|_{A / C}=\varphi$. Hence $\mathcal{B}_{A_{d}^{*}} \cong \mathcal{B}_{C(X)_{d}^{*}}$ and then, by [5],

$$
\operatorname{ext}\left(\mathcal{B}_{A_{d}^{*}}\right)=\operatorname{ext}\left(\mathcal{B}_{C(X)_{d}^{*}}\right)=\left\{\alpha \delta_{x, x^{\prime}}: x, x^{\prime} \in X, x \neq x^{\prime}, \alpha \in \mathbb{T}\right\} .
$$

Remark 3.4. Thanks to Proposition 3.3 , Theorem 3.1 is valid for the fairly large class of algebras which are dense in $\left(C(X),\|\cdot\|_{\infty}\right)$ for a compact space $X$. This class includes, for example, all (real-valued) function algebras on compact spaces and classical function algebras such as the algebra of $n$-times continuously differentiable functions $\left(C^{(n)}(X)\right)$, of Lipschitz functions $(\operatorname{Lip}(X))$, of little Lipschitz functions of order $\alpha \in(0,1)\left(\operatorname{lip}_{\alpha}(X)\right)$, of continuous functions of bounded variation $(B V C(X))$ and of absolutely continuous functions $(A C(X))$ on appropriate compact spaces $X$.

Corollary 3.5. Let $\left(X, d_{1}\right)$ and $\left(Y, d_{2}\right)$ be compact metric spaces and let $T$ : $\operatorname{Lip}(\mathrm{X}) \longrightarrow \operatorname{Lip}(\mathrm{Y})$ be a linear surjective diameter preserving map. Then there 
are a bi-Lipschitz homeomorphism $\psi$ from $Y$ onto $X$, a scalar $\lambda \in \mathbb{T}$ and a linear functional $L: \operatorname{Lip}(\mathrm{X}) \rightarrow \mathbb{C}$ such that for all $f \in \operatorname{Lip}(\mathrm{X}), y \in Y, T f(y)=$ $\lambda f(\psi(y))+L(f)$.

Proof. Let $A=\operatorname{Lip}(X)$ and $B=\operatorname{Lip}(Y)$. Since $A$ and $B$ are dense in $\left(C(X),\|\cdot\|_{\infty}\right)$ and $\left(C(Y),\|\cdot\|_{\infty}\right)$, respectively, Theorem 3.1 and Proposition 3.3 imply that there exist a homeomorphism $\psi: Y \rightarrow X$, a scalar $\lambda \in \mathbb{T}$ and a linear functional $L: A \rightarrow \mathbb{C}$ such that for all $f \in A, y \in Y, T f(y)=\lambda f(\psi(y))+L(f)$.

Let us show that $\psi$ is a Lipschitz map. Define a linear map $T_{0}: A \rightarrow B$ by $T_{0}(f)=f \circ \psi$ for all $f \in A$. Clearly $T_{0}$ is continuous by the Closed Graph theorem. Then, for every $f \in \operatorname{Lip}(X),\|f \circ \psi\| \leq t\|f\|$, where $t=\left\|T_{0}\right\|$. Let $y, y^{\prime}$ be two distinct elements in $Y$. For $x=\psi(y) \in X$ define $f_{x}(z)=d_{1}(x, z)$ for all $z \in X$. Obviously, $f_{x} \in A$ with $\left\|f_{x}\right\| \leq k$, where $k=1+\operatorname{diam}(X)$. Since $T_{0}$ is continuous, $\left\|T_{0} f_{x}\right\| \leq t k$ and, in particular, the Lipschitz constant $L\left(T_{0} f_{x}\right) \leq t k$. On the other hand,

$$
T_{0} f_{x}(y)=f_{x}(\psi(y))=0
$$

and

$$
T_{0} f_{x}\left(y^{\prime}\right)=f_{x}\left(\psi\left(y^{\prime}\right)\right)=d_{1}\left(\psi(y), \psi\left(y^{\prime}\right)\right)
$$

Hence

$$
\frac{d_{1}\left(\psi(y), \psi\left(y^{\prime}\right)\right)}{d_{2}\left(y, y^{\prime}\right)}=\frac{\left|T_{0} f_{x}(y)-T_{0} f_{x}\left(y^{\prime}\right)\right|}{d_{2}\left(y, y^{\prime}\right)} \leq t k .
$$

Therefore, $\sup _{\substack{y, y^{\prime} \in Y \\ y \neq y^{\prime}}} \frac{d_{1}\left(\psi(y), \psi\left(y^{\prime}\right)\right)}{d_{2}\left(y, y^{\prime}\right)} \leq t k$; that is, $\psi$ satisfies the Lipschitz condition on $Y$. Similarly $\psi^{-1}$ is a Lipschitz function on $X$.

We recall that for a metric space $(X, d)$ and $\alpha \in(0,1)$, the algebra $\operatorname{lip}_{\alpha}(X, d)$ (or simply $\operatorname{lip}_{\alpha}(X)$ ) consists of all complex-valued functions $f$ such that $L_{\alpha}(f)=$ $\sup \left\{\frac{\left|f(x)-f\left(x^{\prime}\right)\right|}{d\left(x, x^{\prime}\right)^{\alpha}}: x, x^{\prime} \in X, x \neq x^{\prime}\right\}$ is finite and also $\frac{\left|f(x)-f\left(x^{\prime}\right)\right|}{d\left(x, x^{\prime}\right) \alpha} \rightarrow 0$ when $d\left(x, x^{\prime}\right) \rightarrow 0$.

Remark 3.6. This corollary is true for linear surjective diameter preserving mappings defined between little Lipschitz algebras of the same order. This is not a restriction since, for $0<\alpha<\beta<1, \operatorname{lip}_{\alpha}\left(X, d_{1}\right)=\operatorname{lip}_{\beta}\left(X, d_{2}\right)$, where $\left(X, d_{2}\right)=$ $\left(X, d_{1}^{\frac{\alpha}{\beta}}\right)$. Furthermore, in this context, $\psi$ is a bi-Lipschitz function of the same order.

\section{The Locally Compact Case}

Lemma 4.7. Let $A$ be a function algebra on a locally compact space $X$ and $A_{1}$ its unitization. Then $C h\left(A_{1}\right) \subseteq C h(A) \cup\{\infty\}$. 
Proof. Consider the subalgebra $A_{1}:=A+\mathbb{C} 1$ of $C\left(X_{\infty}\right)$ and let $\overline{A_{1}}$ be the closure of $A_{1}$ in $\left(C\left(X_{\infty}\right),\|\cdot\|_{\infty}\right)$. Fix $\infty \neq x_{0} \in C h\left(\overline{A_{1}}\right)=C h\left(A_{1}\right)$. Let $V$ be a neighborhood of $x_{0}$ in $X$ and let $u \in C_{0}(X)$ be such that $0 \leq u \leq 1$ on $X$, $u\left(x_{0}\right)=1$ and $u=0$ on $X \backslash V$. Set $\alpha=\sup \left\{\operatorname{Re} h\left(x_{0}\right): h \in \overline{A_{1}}, \operatorname{Re} h \leq u\right\}=$ $\sup \left\{\operatorname{Re} h\left(x_{0}\right): h \in A_{1}, \operatorname{Re} h \leq u\right\}$. Since $\overline{A_{1}}$ is a uniform algebra on $X_{\infty}$, then $\alpha=1$ by [11, Lemma 7.19]. So we can take an element $g \in A_{1}$ with $\operatorname{Re} g \leq u$ on $X_{\infty}$ and $\operatorname{Re} g\left(x_{0}\right)>\frac{\ln 14}{\ln 16}$. Then, for $c=\ln 16, f=e^{c(g-1)} \in \overline{A_{1}}$ with $\|f\|_{\infty} \leq 1$, $\left|f\left(x_{0}\right)\right|>\frac{7}{8}$ and $|f| \leq \frac{1}{16}$ on $X_{\infty} \backslash V$. Taking $\lambda=f(\infty)$, we see that $|\lambda| \leq \frac{1}{16}$ and $h=\frac{17(f-\lambda)}{16} \in \bar{A}$ such that $\|h\|_{\infty} \leq 1,\left|h\left(x_{0}\right)\right|>\frac{3}{4}$ and $|h|<\frac{1}{4}$ on $X \backslash V$. Hence $x_{0} \in C h(\bar{A})=C h(A)$ and, therefore, $C h\left(A_{1}\right) \subseteq C h(A) \cup\{\infty\}$.

Note that for a function algebra $A$ on a locally compact space $X, \operatorname{ext}\left(\mathcal{B}_{\left(A_{1}\right)_{d}^{*}}\right)=$ $\left\{\alpha \delta_{x, x^{\prime}}: x, x^{\prime} \in C h\left(A_{1}\right), x \neq x^{\prime}, \alpha \in \mathbb{T}\right\}$ if, and only if, the extreme points of the unit ball of the dual space of $A$ with the diameter norm is the set $\left\{\alpha \delta_{x, x^{\prime}}: x, x^{\prime} \in\right.$ $\left.C h(A) \cup\{\infty\}, x \neq x^{\prime}, \alpha \in \mathbb{T}\right\}$.

Theorem 4.8. Let $A$ and $B$ be two (complex or real-valued) function algebras on locally compact spaces $X$ and $Y$, respectively, such that the set of extreme points of the unit ball of $\left(A_{1}\right)_{d}^{*}$ consists of the set $\left\{\alpha \delta_{x, x^{\prime}}: x, x^{\prime} \in C h\left(A_{1}\right), x \neq\right.$ $\left.x^{\prime}, \alpha \in \mathbb{T}\right\}$. Assume that $T: A \rightarrow B$ is a linear surjective diameter preserving map. Then there are a subset $Y_{0}$ of $C h(B) \cup\{\infty\}$, a continuous injective map $\psi: Y_{0} \rightarrow C h(A) \cup\{\infty\}$, a scalar $\lambda \in \mathbb{T}$ and a linear functional $L: A \rightarrow \mathbb{C}$ such that for all $f \in A, y \in Y_{0}, T f(y)=\lambda f(\psi(y))+L(f)$.

Proof. Since by defining $f(\infty)=0$ for each $f \in A$, we can consider $f$ as a member in $C\left(X_{\infty}\right)$, then $A$ can be regarded as an ideal in $A_{1}=A+\mathbb{C} 1 \subseteq C\left(X_{\infty}\right)$. Let us recall here that $A_{1_{d}}$ and $B_{1_{d}}$ denote the quotient spaces $A_{1} / C$ and $B_{1} / C$, respectively. Thus, $S: A_{1_{d}} \rightarrow B_{1_{d}}$ defined by $S(\pi(f+\gamma))=\pi(T f+\gamma)$ for all $f \in A$ and $\gamma \in \mathbb{C}$, is a linear isometry under the diameter norm. To see this, take $f \in A$ and $\gamma \in \mathbb{C}$. Then

$$
\begin{aligned}
\operatorname{diam}(S(\pi(f+\gamma))) & =\sup _{y, y^{\prime} \in Y}\left|S(\pi(f+\gamma))(y)-S(\pi(f+\gamma))\left(y^{\prime}\right)\right| \\
& =\sup _{y, y^{\prime} \in Y}\left|T f(y)-T f\left(y^{\prime}\right)\right|=\sup _{x, x^{\prime} \in X}\left|(f+\gamma)(x)-(f+\gamma)\left(x^{\prime}\right)\right| \\
& =\sup _{x, x^{\prime} \in X}\left|\pi(f+\gamma)(x)-\pi(f+\gamma)\left(x^{\prime}\right)\right|=\operatorname{diam}((\pi(f+\gamma)) .
\end{aligned}
$$

Since $B$ is a function algebra, $\left\{\delta_{y}: y \in C h(B) \cup\{\infty\}\right\}$ is linearly independent in the dual space of $B_{1}$. Then, by an argument similar to the one used in the proof of Theorem 3.1 for the map $S$, we obtain a subset $Y_{0}$ of $C h(B) \cup\{\infty\}$, a continuous injective map $\psi: Y_{0} \rightarrow C h(A) \cup\{\infty\}$ and a scalar $\lambda \in \mathbb{T}$ such that for all $f \in A$ and $y, y^{\prime} \in Y_{0}, T f(y)-T f\left(y^{\prime}\right)=\lambda\left(f(\psi(y))-f\left(\psi\left(y^{\prime}\right)\right)\right)$. In particular, if $\psi\left(y^{\prime}\right)=\infty$, then we have $T f(y)=\lambda f(\psi(y))+T f\left(y^{\prime}\right)$ for all $f \in A$ and $y \in Y_{0}$. 
Now, if we define a linear functional $L: A \rightarrow \mathbb{C}$ by $L(f)=T f\left(y^{\prime}\right)$ for all $f \in A$. Then, for each $f \in A$ and $y \in Y_{0}, T f(y)=\lambda f(\psi(y))+L(f)$.

Remark 4.9. In the locally compact case, $\psi$ does not always leave the infinity fixed. For example, let $X=(0,1]$ and $Y=\left[0, \frac{1}{2}\right) \cup\left(\frac{1}{2}, 1\right]$. Then $X$ and $Y$ are not homeomorphic, however, their one point compactifications coincide. Now, it is not difficult to define a linear diameter preserving map $T$ from $C_{0}(X)$ onto $C_{0}(Y)$ which is not an isometry under the supremum norm (see [6, Remark 18]). Hence the homeomorphism induced by the map $T$ cannot take the infinity to the infinity. See more examples in [6].

Question: Is there a linear surjective diameter preserving mapping defined between two function algebras which cannot be written as the sum of a weighted composition operator and a linear functional on their Choquet boundaries?

\section{ACKNOWLEDGMENT}

This paper was conceived while the second-named author enjoyed the hospitality of the Department of Mathematics of Universitat Jaume I (Spain) in 2009.

\section{REFERENCES}

1. A. Aizpuru and F. Rambla, There's something about the diameter, J. Math. Anal. Appl., 330 (2007), 949-962.

2. A. Aizpuru and M. Tamayo, Linear bijections which preserve the diameter of vectorvalued maps, Linear Algebra Appl., 424 (2007), 371-377.

3. A. Aizpuru and M. Tamayo, On diameter preserving linear maps, J. Korean Math. Soc., 45 (2008), 197-204.

4. B. Barnes and A. Roy, Diameter preserving maps on various classes of function spaces, Studia Math., 153 (2002), 127-145.

5. F. Cabello Sánchez, Diameter preserving linear maps and isometries, Arch. Math., 73 (1999), 373-379.

6. J. J. Font and M. Sanchis, A characterization of locally compact spaces with homeomorphic one-point compactifications, Topology Appl., 121 (2002), 91-104.

7. J. J. Font and M. Sanchis, Extreme points and the diameter norm, Rocky Mountain J. Math., 34 (2004), 1325-1331.

8. F. González and V. V. Uspenskij, On homomorphisms of groups of integer-valued functions, Extracta Math., 14 (1999), 19-29.

9. M. Györy and L. Molnár, Diameter preserving linear bijections of $C(X)$, Arch. Math., 71 (1998), 301-310. 
10. T. S. S. R. K. Rao and A. K. Roy, Diameter preserving linear bijections of function spaces, J. Aust. Math. Soc., 70 (2001), 323-335.

11. E. L. Stout, The theory of uniform algebras, Bogden and Quigley, Inc. Publishers, 1971.

Juan J. Font

Departamento de Matemáticas

Universitat Jaume I

Campus Riu Sec.

8029 AP, Castellón

Spain

E-mail: font@mat.uji.es

Maliheh Hosseini

Department of Mathematics

Tarbiat Modares University

Tehran, Iran

E-mail: hosseini_m@modares.ac.ir 
